a history as a list of references from which a history of the theory of numbers might be written. Be that as it may, there is the greatest need for just such a piece of work to promote efficiency among the professional workers in this field and to prevent them from wasting their time on problems that have already been adequately treated, and also to suggest other problems which still defy analysis. It is to be hoped that the second volume will not be long delayed.

D. N. LehMer.

\title{
THE CALCULUS OF PROBABILITY.
}

Calcolo delle Probabilità. By Guido Castelnuovo. Albrighi, Segati \& C., Milano-Roma-Napoli, 1919. xxiii + 373 pp.

THE increased interest in the calculus of probability which has arisen during the past fifty years has been due in no small part to the brilliant applications of it in the field of physical phenomena. One of the most important of these, first in point of time and a model for the others, is due to Maxwell and has to do with the distribution of velocities of the molecules of a gas. Some of these investigations of physical phenomena on the basis of the laws of probability, operating under an assumed absence of determining physical laws among certain groups of phenomena, have been so successful in accounting for or predicting physical events that the conception has arisen in some quarters of the "laws of nature" as merely certain statements of average among fortui ous occurrences. It is almost uncanny to find relatively constant results of measurements of certain sorts predicted by a mathematical analysis based essentially on an assumption of chance distribution; and yet this is found in not a few important investigations.

A paradoxical situation of this sort will always excite interest. The human mind is peculiarly uncomfortable in the presence of a demonstrated result and an intuitive feeling between which there seems to be disharmony. A disturbance of our equilibrium is produced when we see the theory of probability thus accounting for what seemed to be fixed relations among phenomena. Where there is lack of equilibrium there is 
resiless thought; and in the presence of the latter new analyses are made of the established results of science relevant to the questions raised. This perhaps accounts in some measure for the large number of treatises on the theory of probability published in recent years.

It is interesting to observe that in the field where these physical applications were first made and where they have been most successful perhaps in relating physical phenomena by means of theoretical investigations there is yet a feeling of dissatisfaction with the situation from the critical point of view of rigorous thinking. A minute examination of the various demonstrations proposed for a justification of Maxwell's law of distribution of velocities brings out the fact that the reasoning, though meriting the attention which it has received and in its first aspects appearing persuasive if not conclusive, is nevertheless not immune to criticism. In fact there is sufficient doubt concerning the logical processes, in any of the several proofs advanced, to leave one with a desire for some additional support-so much so, that a frank and conscientious statement would probably bring out the confession that one is somewhat relieved by the fact of the success of the theory in accounting for physical phenomena.

This most noteworthy instance which arises in the applications may serve to emphasize the oft-mentioned fact that great logical difficulties are present in the whole theory of probability. It manifests itself from the outset, being present to start with in the definition of probability. The difficulty has so persisted here, in spite of all the careful analysis which has been made and the varying form of definition which has been proposed, that one cannot yet avoid a feeling of discomfort from the start. In a range of ideas having no contact with other things and no applications in other fields, such a feeling of discomfort would do much to inhibit a detailed development of theory; one would be disposed to labor longer to clear up the initial logical difficulties. But in this case there are so many points of contact with other interests and during the past fifty years such an increasing suggestiveness of fundamental value in physical investigations that one is disposed to lay aside temporarily his feeling of discomfort and to continue to proceed tentatively with the theory till he is able to check it up through a testing of the results.

When one proceeds thus in a range of ideas somewhat 
lacking in clarity and in logical precision, it is peculiarly important that the significance of the initial ideas, the character of the difficulties, and the steps of the argument, shall be rendered as clear as possible through a suitable exposition. The author of the present treatise is particularly successful in making clear at the beginning of each section the problem which is to be treated in that section and then in summing up his results clearly at the proper place. Moreover the more difficult or more elusive notions are treated with care and the learner is not only presented with a correct statement but is also warned against certain probable errors. Illustrative cases are well chosen for bringing out clearly the character of the notions in consideration. If all exposition maintained in this respect the same felicity as this book, students of mathematics would be saved a great deal of energy not infrequently diverted from a mastery of the matter by the necessity of an undue effort to follow the author's form or order of presentation.

Several subjects of mathematics, depending on a minimum of technical information and detail though requiring maturity for their mastery (like the calculus of probability), would become accessible to a wider range of readers interested in science if presented in such way as to be more readily understood. Such persons, if they attempted to read the current expositions, would now too often be repelled by the forbidding style of a condensed exposition, frequent in technical mathematical works, which wastes much more energy and of greater value than that which would be expended in printing the somewhat larger volumes needed for an exposition more carefully serving the reader's comfort. It is poor economy when a little printer's ink and paper are saved at the expense of an increase in the expenditure of scientific energy needed to read an exposition which has been too much condensed through improper omissions.

Two means are used in the present work to keep the main body of the book accessible to readers with a minimum mathematical training: a considerable part of the more technical matter is put in the appendix of sixty-four pages; a few articles are marked with an asterisk and these may be omitted without destroying continuity. The latter sometimes give new results; at other times they put on a more rigorous or more exact basis what had already been developed in a more intuitive way and 
with less care for completeness. An instance of the latter sort is afforded by sections 45 and 46, where one has a more satisfactory derivation and discussion of certain approximations earlier gotten in a somewhat formal way. The less mature reader may omit these more critical sections; he will perhaps not be dissatisfied with the earlier work, where approximate formulas are used rather too much as exact formulas are employed.

About half of the nearly three hundred pages in the main body of the work is devoted to the more elementary and more general aspects of the theory. Here the exposition is particularly marked by that clarity of expression which is characteristic of the entire work. The reader is led in a pleasing way through such topics as probability and frequency, total and composite probability, mathematical expectation and mean value, the problem of repeated trials and the theorem of Bernoulli, the normal law of probability, and probability involving continuous distribution. Then one meets in the second half of the text brief chapters on probability of causes, statistical interpretation, errors of observation, method of least squares, and the kinetic theory of gases. The greater portion of the appendix is given to a careful consideration of the theorem of Laplace-Tchebychef and an extension of it.

R. D. Carmichael.

\section{CORRECTIONS.}

The following errata have come to the attention of the editors of the Bulletin:

In Professor Brouwer's paper: "Intuitionism and formalism," in volume 20 , pages $81-96$,

page 91, in the footnote, for: the proof read: the formalistic proof of this property; and interchange "können wir bestimmen" with "muss es geben;"

page 94, line 4, for: but that it is impossible read: and when a proof is added (for the rest not recognized by the intuitionist) that it is impossible.

In Professor Forsyth's paper: "Formulas for constructing abridged mortality tables for decennial ages," in the current volume, 